\title{
4. SCALE OF CHORDS
}

With a scale of chords (figure 29) it is possible to construct angles without the use of a protractor. To construct the scale:

1. On base line $0-b$ draw a quadrant.

2. Divide the quarter circle into 18 equal parts and number them $0-90$ as shown.

3. With centre 0 draw arcs from all the points on the curve to terminate on line $0-b$.

4. Using these points draw the scale immediately below the quadrant as shown. (A piece of thin plywood or similar flat material should be obtained and the scale carefully marked on the plywood.)
To construct the triangle $a-b-c$ (figure 30 ) using the scale of chords:

1. Draw the base line $a-b$.

2. With radius $0-60$ (from the scale) and with centres $a$ and $b$ in turn, draw the two arcs.

3. With centre $x$ and radius $0-50$, mark off the point $d$. Draw a $-\mathrm{d}$ to construct an angle of $50^{\circ}$.

4. With centre $y$ and radius $0-35$ mark off the point $e$ to construct an angle of $35^{\circ}$. The two lines meet at $c$. $a-b-c$ is the required triangle

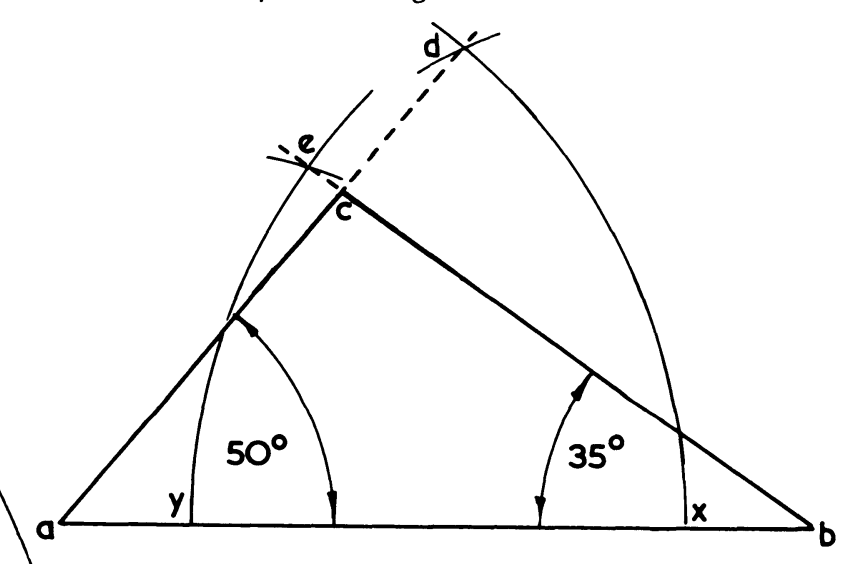

Figure 30

Figure 29
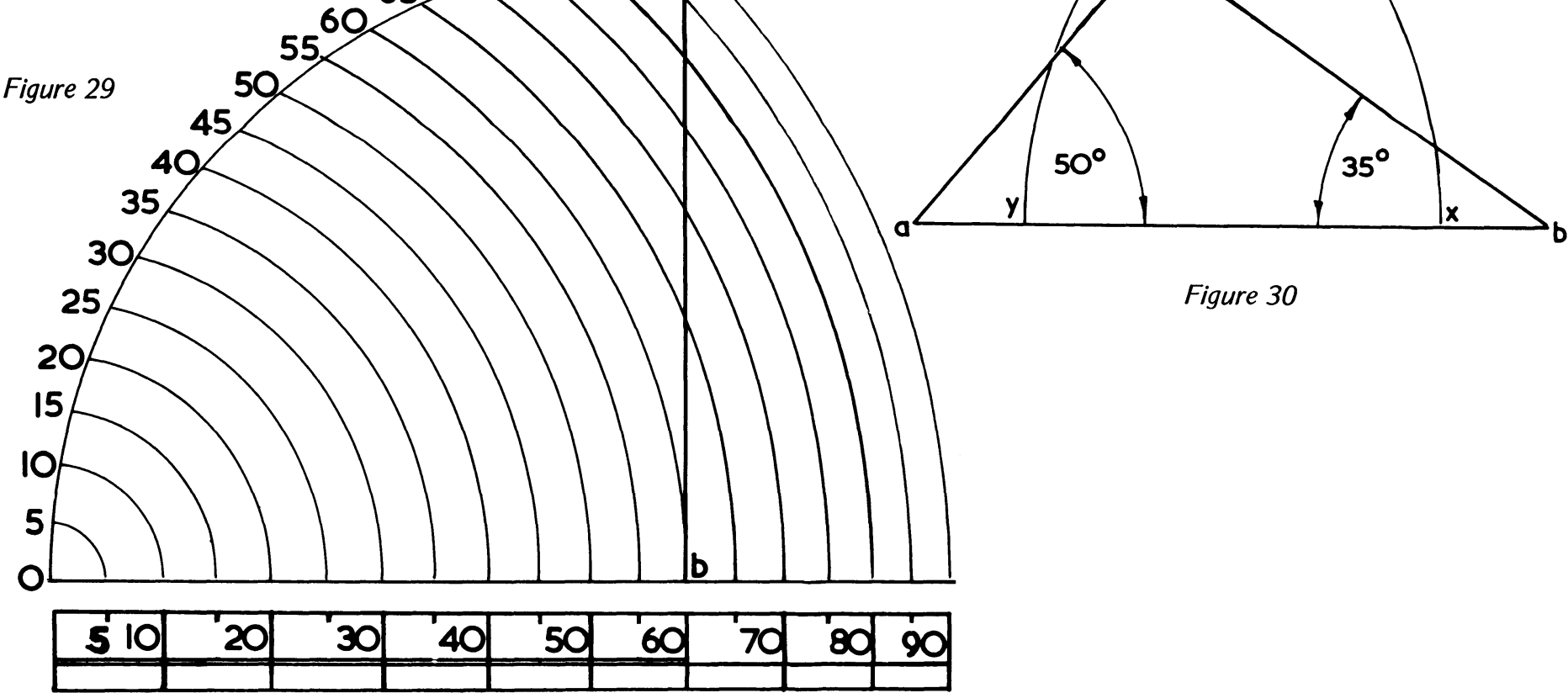